\title{
High-Energy X-ray Diffraction and Topography Investigation of CdZnTe
}

\author{
G.A. CARINI, ${ }^{1,2,5}$ G.S. CAMARDA, ${ }^{1}$ Z. ZHONG ${ }^{3}$ D.P. SIDDONS, ${ }^{3}$ \\ A.E. BOLOTNIKOV,${ }^{1}$ G.W. WRIGHT, ${ }^{1}$ B. BARBER,${ }^{4}$ C. ARNONE, ${ }^{2}$ \\ and R.B. JAMES ${ }^{1}$
}

1.-Brookhaven National Laboratory, Upton, NY 11973. 2.-Department of Electrical Engineering, University of Palermo, 90128 Palermo, Italy. 3.-National Synchrotron Light Source, Brookhaven National Laboratory. 4.-University of Arizona, Tucson, AZ, 85721. 5.-E-mail: carini@bnl.gov

High-energy transmission $\mathrm{x}$-ray diffraction techniques have been applied to investigate the crystal quality of CdZnTe (CZT). CdZnTe has shown excellent performance in hard x-ray and gamma detection; unfortunately, bulk nonuniformities still limit spectroscopic properties of CZT detectors. Collimated high-energy x-rays, produced by a superconducting wiggler at the National Synchrotron Light Source's X17B1 beamline, allow for a nondestructive characterization of thick CZT samples (2-3 mm). In order to have complete information about the defect distribution and strains in the crystals, two series of experiments have been performed. First, a monochromatic $67 \mathrm{keV}$ x-ray beam with the size of $300 \times 300 \mu \mathrm{m}^{2}$ was used to measure the rocking curves of CZT crystals supplied by different material growers. A raster scan of a few square centimeter area allowed us to measure the full-width at half-maximum (FWHM) and shift in the peak position across the crystal. The rocking curve peak position and its FWHM can be correlated with local stoichiometry variations and other local defects. Typically, the FWHM values ranging from 8.3 arcsec to 14.7 arcsec were measured with the best crystal used in these measurements. Second, transmission white beam x-ray topography (WBXT) was performed by using a $22 \mathrm{~mm} \times 200 \mu \mathrm{m}$ beam in the energy range of $50 \mathrm{keV}$ to $200 \mathrm{keV}$. These types of measurements allowed for large area, high-resolution $(50 \mu \mathrm{m})$ scans of the samples. Usually, this technique is used to visualize growth and process-induced defects, such as dislocations, twins, domains, inclusions, etc. the difference in contrast shows different parts of the crystal that could not be shown otherwise. In topography, good contrast is indicative of a high quality of the sample, while blurred gray shows the presence of defects. Correlation with other techniques (e.g., infrared (IR) mapping and gamma mapping) was also attempted. Our characterization techniques, which use highly penetrating x-rays, are valid for in-situ measurements, even after electrical contacts have been formed on the crystal in a working device. Thus, these studies may lead to understanding the effects of the defects on the device performance and ultimately to improving the quality of CZT material required for device fabrication. It is important to study crystals from different ingot positions (bottom, center, and top); consequently, more systematic studies involving scans from center to border are planned.

Key words: High-energy x-ray diffraction, rocking curve, topography, CZT, crystal quality, detectors characterization 


\section{INTRODUCTION}

$\mathrm{CZT}^{1,2}\left(\mathrm{Cd}_{1-\mathrm{x}} \mathrm{Zn}_{\mathrm{x}} \mathrm{Te}\right)$ is an attractive material for the fabrication of high-energy detectors. Its large band gap $\left(\mathrm{E}_{\mathrm{gap}} \cong 1.6 \mathrm{eV}\right)$ allows it to operate at room temperature, and the high atomic numbers of the elements composing the material $\left(\mathrm{Z}_{\mathrm{Cd}}=48, \mathrm{Z}_{\mathrm{Zn}}=30\right.$, and $\mathrm{Z}_{\mathrm{Te}}=52$ ) give a high quantum efficiency. In principle, detectors are simple devices that directly convert charge pairs generated by ionizing radiation, such as x-rays, gamma rays, and beta particles, into electrical signals. These advantages combined with very good resolution make CZT detectors suitable for a wide range of applications such as nuclear weapons monitoring, imaging devices for medical, and astronomy applications.

Detector grade material has a composition of $\mathrm{x}=$ $0.04-0.2$, a resistivity $\rho \approx 10^{10} \Omega \mathrm{cm}$, and mobilitylifetime product for electrons $\mu_{\mathrm{e}} \tau_{\mathrm{e}} \approx 10^{-3} \mathrm{~cm}^{2} / \mathrm{V}$ and for holes $\mu_{\mathrm{h}} \tau_{\mathrm{h}} \approx 10^{-5} \mathrm{~cm}^{2} / \mathrm{V}$.

Historically, the use of the high-pressure Bridgman $^{3}$ technique for CZT crystal growth led to the production of material for larger area detectors. Unfortunately, structural defects such as twins, dislocations, inclusions, grain, and tilt boundaries result in a low crystalline quality and limit the detector's performances. ${ }^{4,5}$

High-energy transmission x-ray diffraction techniques ${ }^{6-9}$ provide a powerful way to study bulk properties of thick crystals (millimeters). As detectors are directly fabricated using a crystal slab, this investigation is suitable to determine the relationship between structural defects and detector response. Highly penetrating x-rays are used for in-situ measurements, even after electrical contacts have been formed on the crystal in a working device. The energy used for these measurements makes reliable the simultaneous measurement of diffraction and detector response. These studies may ultimately lead to the improved quality of CZT material required for device fabrication. ${ }^{10}$

\section{EXPERIMENT}

The X17B1 beamline at BNL's National Synchrotron Light Source provides a collimated highenergy x-ray beam produced by a superconducting wiggler. The experimental hutch was setup for two series of experiments using transmission diffraction techniques. In both experiments, samples, in their holder, were mounted in two translation (x-y) stages located in the center of the triple axis (Fig. 1a and b).

First, a monochromatic $67 \mathrm{keV}$ x-ray beam with the size of $300 \mu \mathrm{m} \times 300 \mu \mathrm{m}$ was used to measure the rocking curves of CZT crystals. A sagittal focusing double Laue monochromator ${ }^{11,12}$ permits a highly collimated beam with a divergence of $10^{-5}$ rad and a flux of $10^{11}$ photons/sec. To record rocking curves, a detector is positioned in a reflection direction, previously determined from the diffraction pattern, and the sample is finely rotated around an angle that meets the Bragg's condition, $\lambda=$

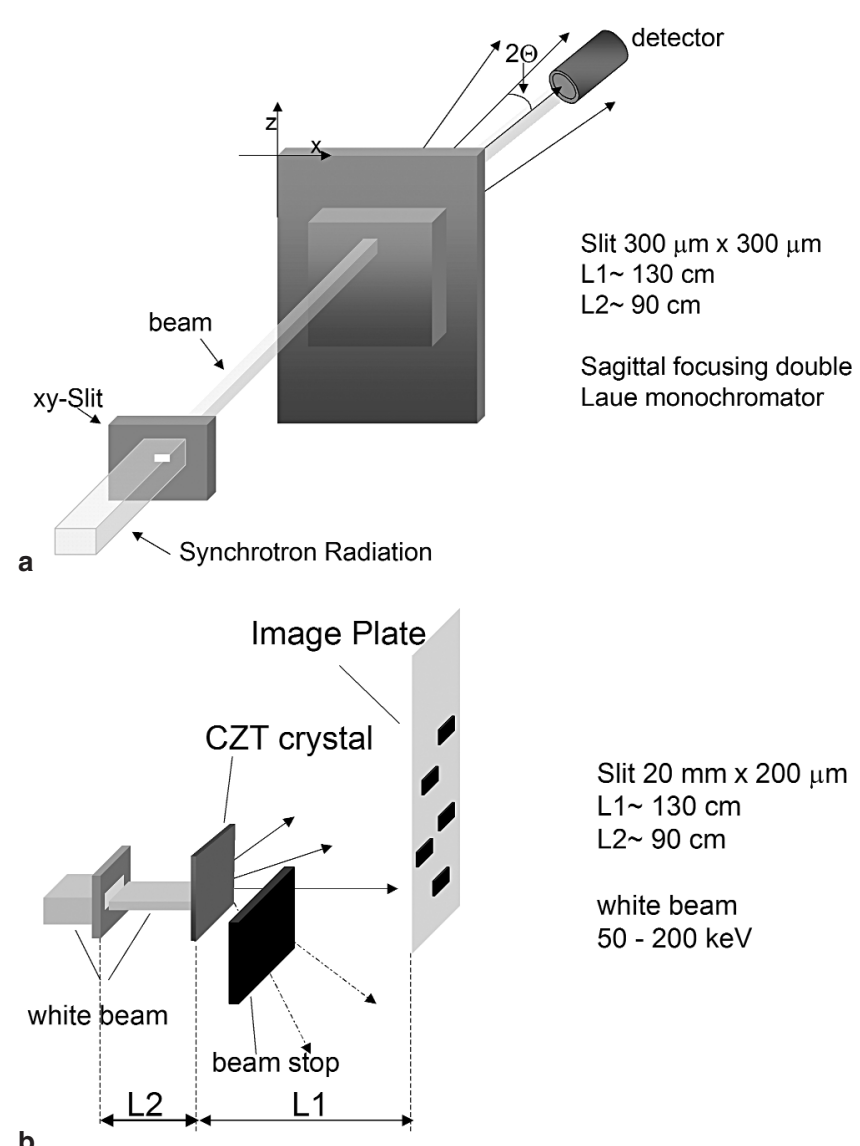

Fig. 1. Experimental setup: (a) rocking curve measurements and (b) WBXT.

$2 \mathrm{~d}_{\mathrm{hkl}} \sin v_{\mathrm{B}}$, where $\lambda$ is the $\mathrm{x}$-ray wavelength $(0.185$ $\AA / 67 \mathrm{keV}), \mathrm{d}_{\mathrm{hkl}}$ is the lattice plane spacing, and $\theta_{\mathrm{B}}$ is the Bragg angle. Thus, this small angle variation makes it possible to register the angular distribution of diffraction spot intensity without losing the reflection direction. The availability of the two stages allows scanning the crystal, mapping point by point with the selected beam size. The typical step of a raster scan is $1 \mathrm{~mm}$ for each direction. As mentioned above, this technique is used to investigate bulk properties; then, this map contains information about the full thickness corresponding to the irradiated area.

Second, transmission white beam x-ray topography (WBXT) was performed by using a $22 \mathrm{~mm} \times 200$ $\mu \mathrm{m}$ beam in the energy range of $50-200 \mathrm{keV}$. A WBXT image, topograph is obtained by projecting the distribution of diffracted intensity produced by a area-filling x-ray beam incident on a sample, onto a film, or an image plate. This is a powerful and simple technique. The basic setup is almost the same used in 1912 for the first x-ray diffraction experiment (Laue technique) with the unique capability that synchrotron radiation offers in terms of a low divergent, homogeneous, and bright beam. Then, short exposure times, between milliseconds and seconds, are required to record a white beam topograph. Moreover, the small ratio between the 
source dimension and the source distance allows a high-resolution scan of the sample.

For this experiment, an image plate, $20 \mathrm{~cm} \times 25 \mathrm{~cm}$ with a resolution of $50 \mu \mathrm{m}$, is positioned far enough from the sample, to reduce background. Both the image plate and sample are translated simultaneously, in the same direction, with a synchronized movement to scan a large area of the crystal.

A SPEC ${ }^{13}$ macro (a UNIX-based software package for instrument control and data acquisition developed for x-ray diffraction) controls $x-y$ stages, triple-axis movements, and data acquisition.

\section{RESULTS AND DISCUSSION}

High-energy transmission x-ray diffraction techniques have been successfully applied to investigate thick CZT samples thanks to the low linear absorption coefficient at these energies (Fig. 2). We investigated samples grown by either modified vertical Bridgman or high-pressure Bridgman techniques, provided by different growers. Sample thicknesses range between $2 \mathrm{~mm}$ and $5 \mathrm{~mm}$.

The CZT has a cubic zincblende crystal structure and a lattice constant between $6.27 \AA$ and $6.43 \AA$ was measured from the diffraction pattern. These values are in agreement with the calculated values for the alloy CdTe-ZnTe (6.48 $\AA$ and $6.10 \AA$, respectively), with compositions in the previously mentioned range.

We probed (422) and (211) rocking curves of the (111) oriented CZT crystals. Full-width at half-maximum (FWHM) and peak position from data Gaussian fitting were used as crystalline quality indexes. The rocking curve of a perfect crystal is a sharp peak (Fig. 3). A mosaic crystal with small regions (compared with the beam size), slightly tilted, produces a broader rocking curve. Distinct peaks arise from a low-angle tilt boundary. Thus, the rocking curve peak position and its FWHM can be correlated with local stoichiometry variations and other local

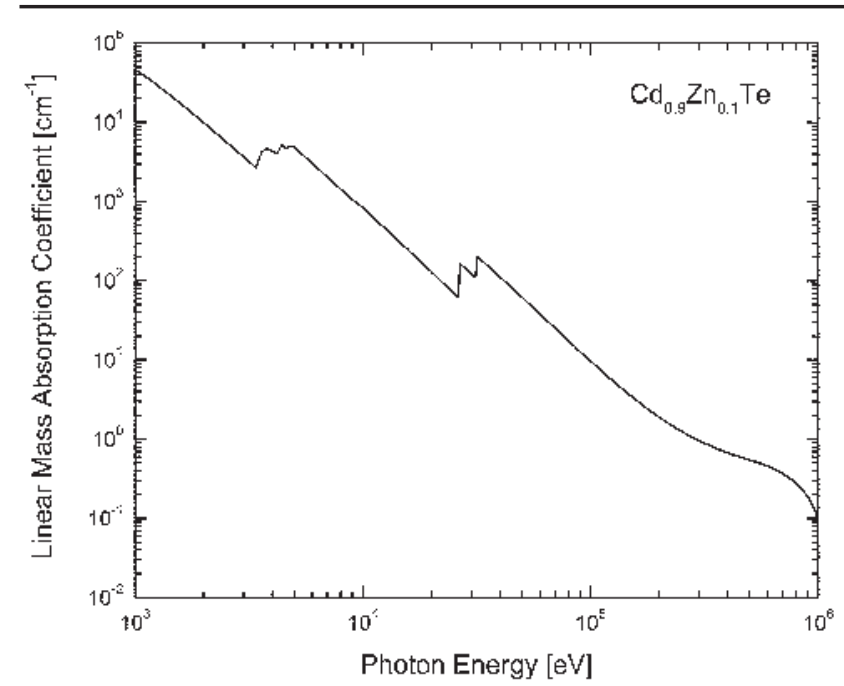

Fig. 2. Linear absorption coeffecient for a typical $\mathrm{Zn}$ concentration. The calculation was performed using $x$-ray oriented programs, http://www.esrf.fr/computing/scientific/xop/).

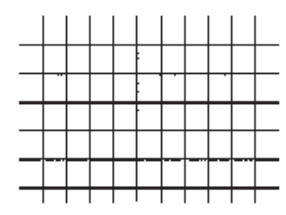

a)

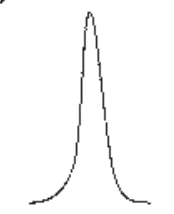

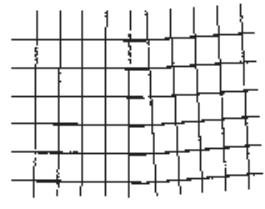

b)

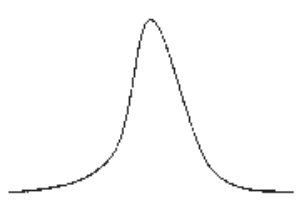

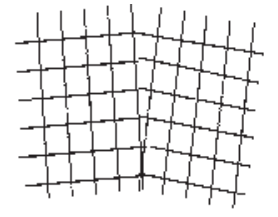

c)

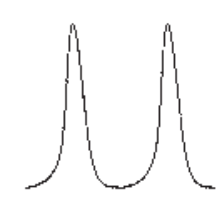

Fig. 3. Schematic diagram of crystalline structure and related rocking curve: (a) perfect crystal, (b) mosaic spread, and (c) tilt boundary.

defects $^{5}$ such as change of lattice constant (variation in $\mathrm{Zn}$ concentration), tilt between macrograins and lattice bending, and slight misorientation in micrograins on the spatial homogeneity.

A raster scan across the crystal allows mapping crystalline quality as a function of position through a series of rocking curves. We scanned, with 1-mm step and $300 \mu \mathrm{m} \times 300 \mu \mathrm{m}$ beam size, an area of a few square centimeters achieving FWHM and peak position maps (Fig. 4). Typically, FWHM values ranging from 8.3 arcsec to 14.7 arcsec were measured for the best crystal used in these runs (Fig. 5).

This type of measurement is time expensive. However, with a faster scan performed with a larger beam, it is difficult to obtain a single peak with low FWHM all over a larger area. Figure 4 exhibits FWHM and peak position shifts in a few millimeters even for the best crystal investigated. On the other hand, a smaller beam permits correlating these measurements with spectroscopic analysis, allowing the evaluation of the local $\mathrm{Zn}$ composition ( $\mathrm{Zn}$ to $\mathrm{Cd}$ ratio can vary radially in the crystal due to thermal gradients), and with infrared (IR) mapping.

Large area and high-resolution images of the samples were performed by transmission WBXT. White beam x-ray topography is used to provide a picture of defect distribution in a crystal. This visualization is possible because of the nonhomogeneous intensity distribution in the image (contrast) arising from the distortion and strain fields. The difference in contrast shows different parts of the crystal that could not be shown otherwise. In topography, good contrast is indicative of a high quality of the sample, while blurred gray shows the presence of defects. This technique visualizes growth and process-induced defects, such as dislocations, twins, domains, and inclusions, even if the interpretation of the topographs is not trivial.

Two fundamental mechanisms can be identified for contrast: orientation contrast and extinction contrast. When a misorientation is present in the investigated area of the crystal (Fig. 6), the beams from the two regions have different directions. The diffracted beam yields bright and dark regions in the recording film, being the first corresponding to a loss 

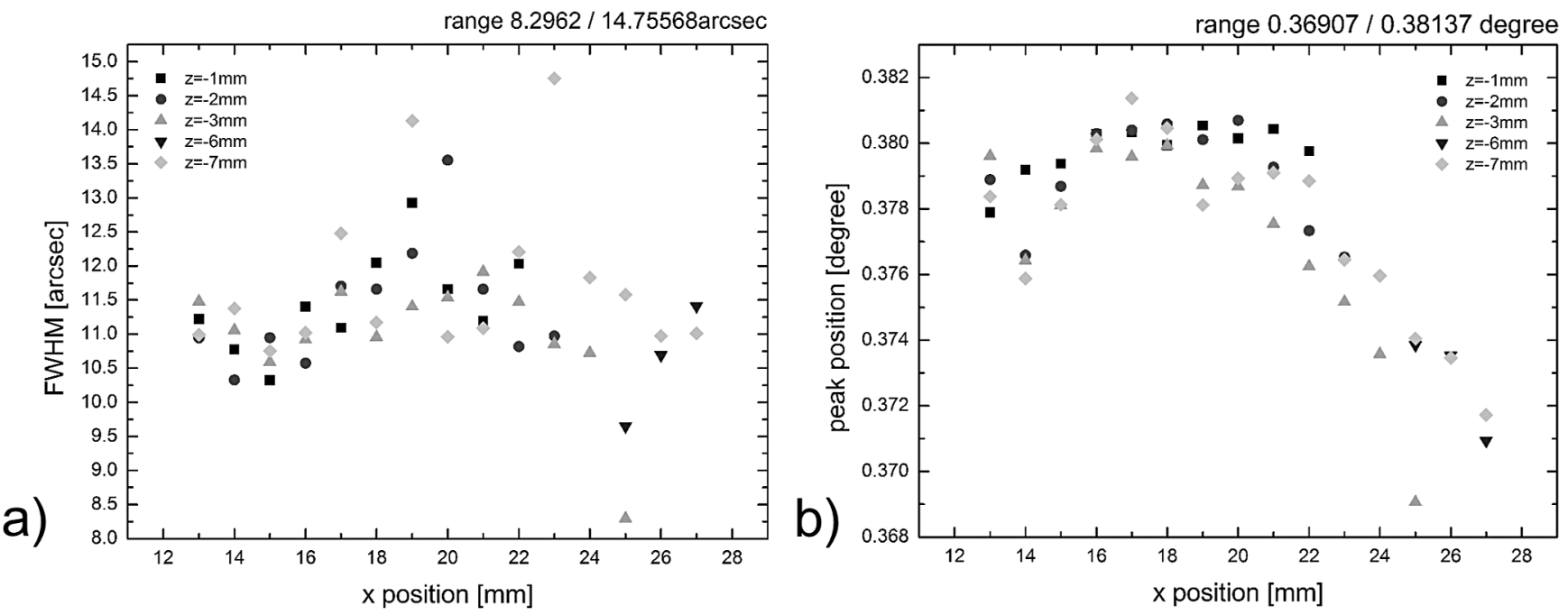

C)
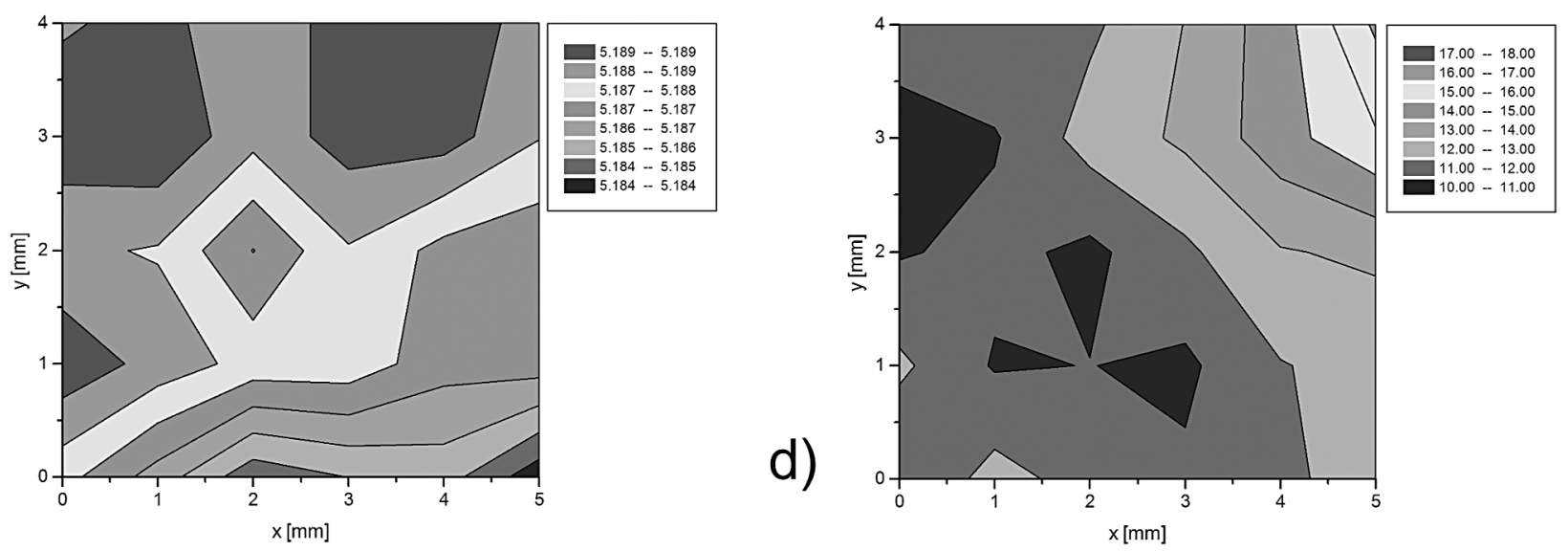

Fig. 4. Peak position and FWHM of a mapped area: (a) and (b) one-dimensional graphs; and (c) and (d) two-dimensional maps.
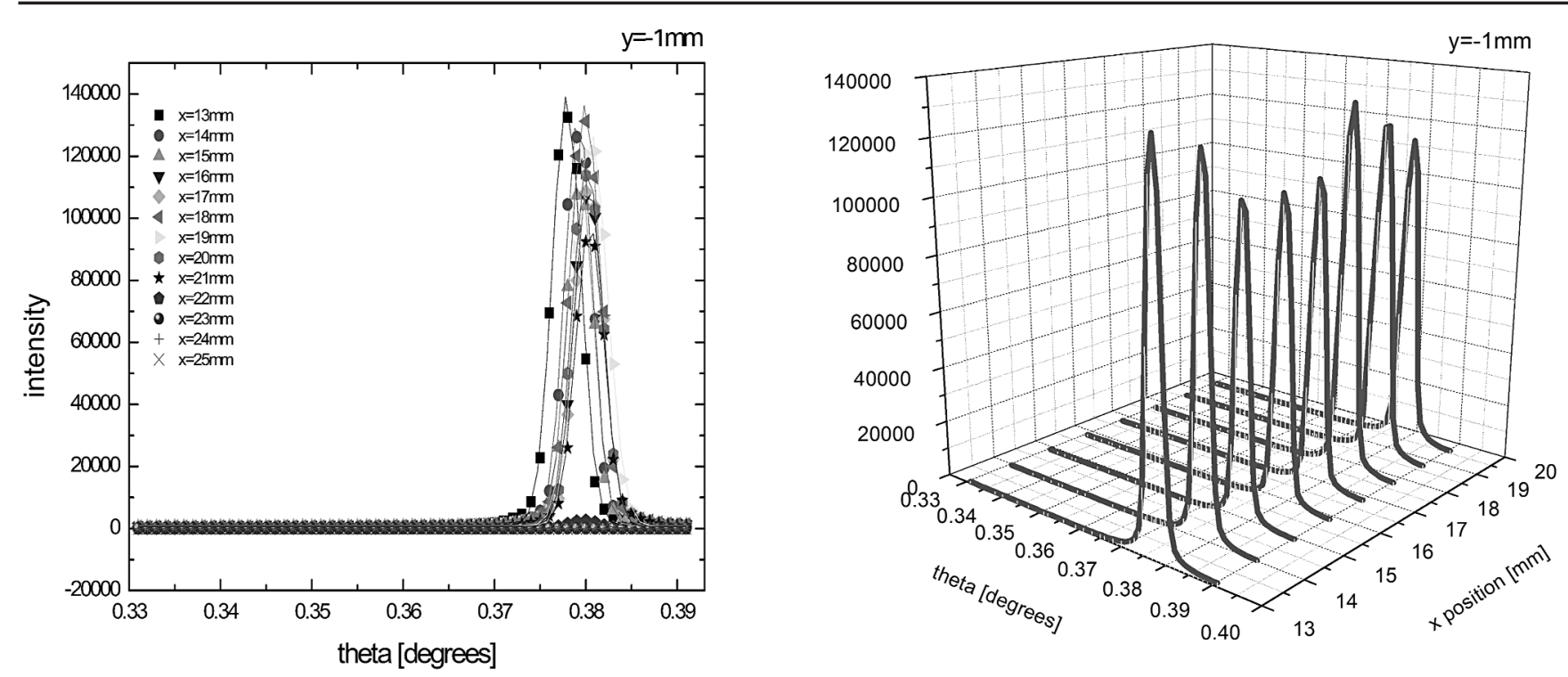

Fig. 5. Rocking curves at a fixed y position scanning $x$ direction.

of ray and the second to a gain. That leads to contrast that appears differently, darker or brighter area, depending on the relation between the misorientation and the source characteristics such as angular source size and spectral width. This orientation contrast can be completely recorded if the distance between the 


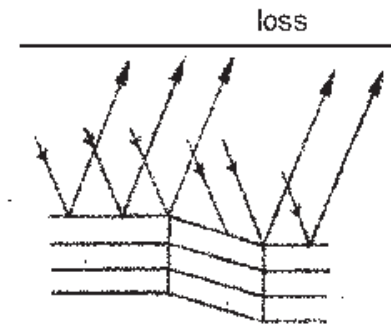

a)

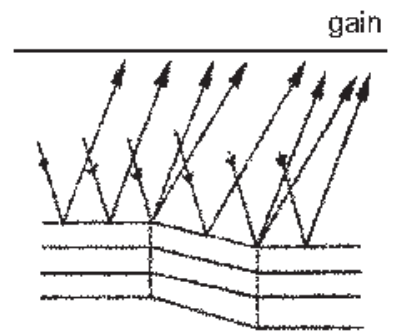

b)

Fig. 6. Schematic diagram of orientation contrast formation: (a) decreased and (b) increased intensity.

sample and the image plate is large enough (Fig. 7). It allows the recognition of twins and subgrain boundaries and thus the number of domains and their distribution in the crystal.

The distortion of the lattice around a defect, dislocation, or precipitate yields different diffraction conditions resulting in different scattered intensity (extinction contrast). This type of contrast in our studies is hard to recognize because of the thickness of the samples.

Then, WBXT is a simple and effective technique allowing the study of several reflections and the

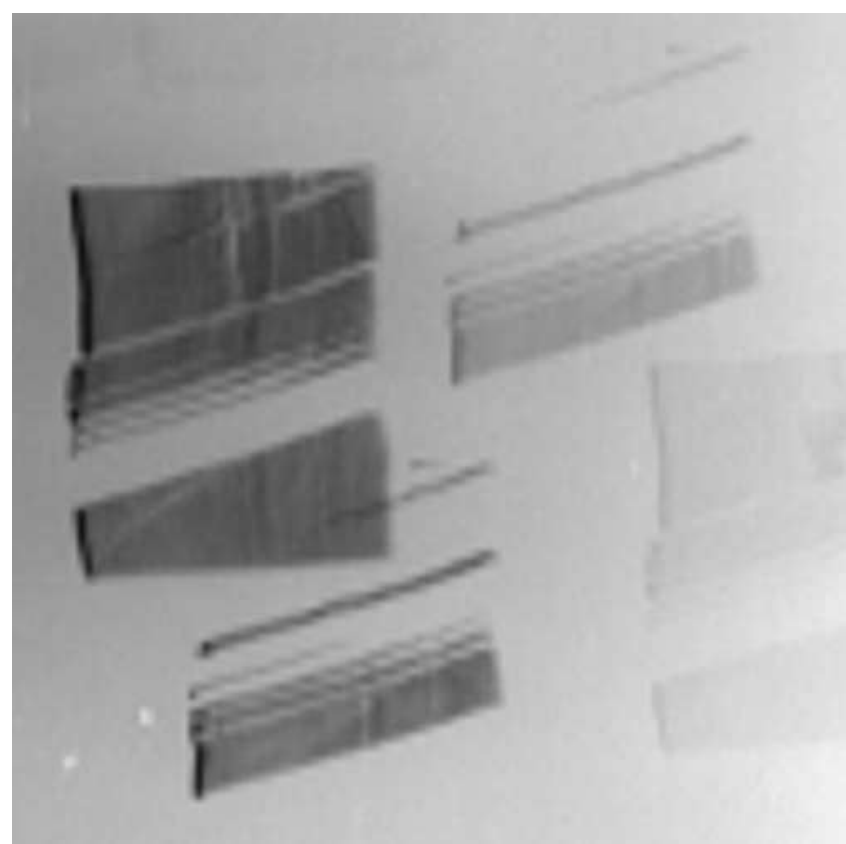

Fig. 7. WBXT, twins details.
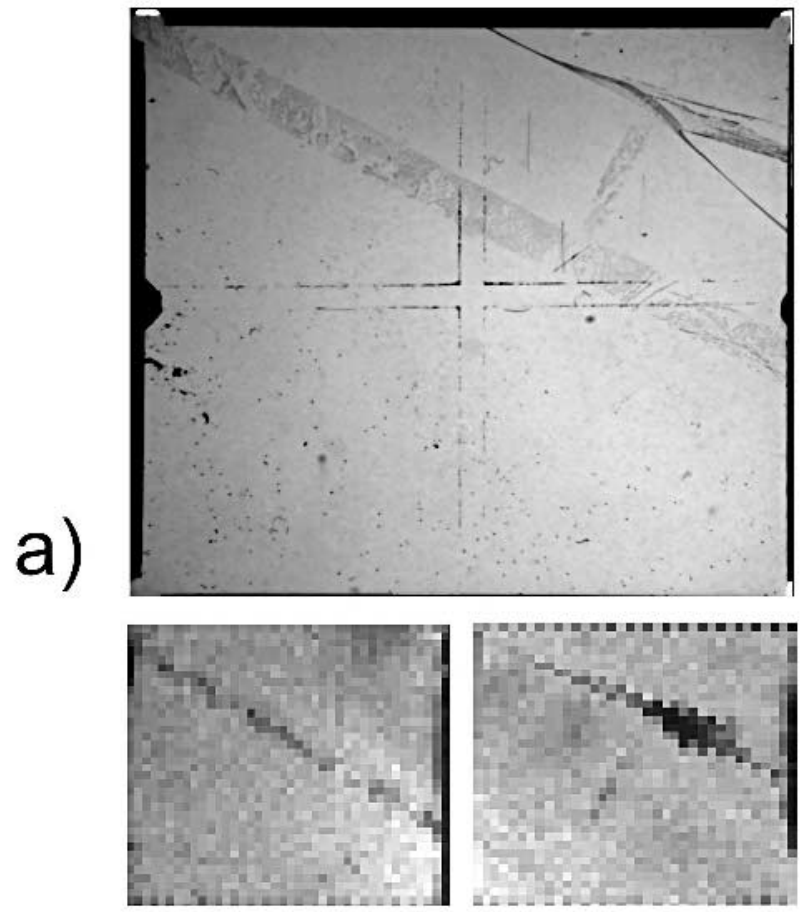

b)
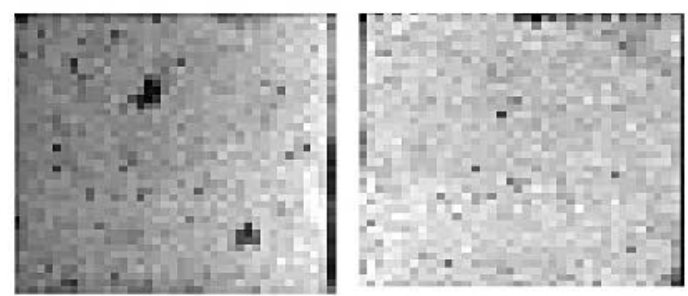

c)

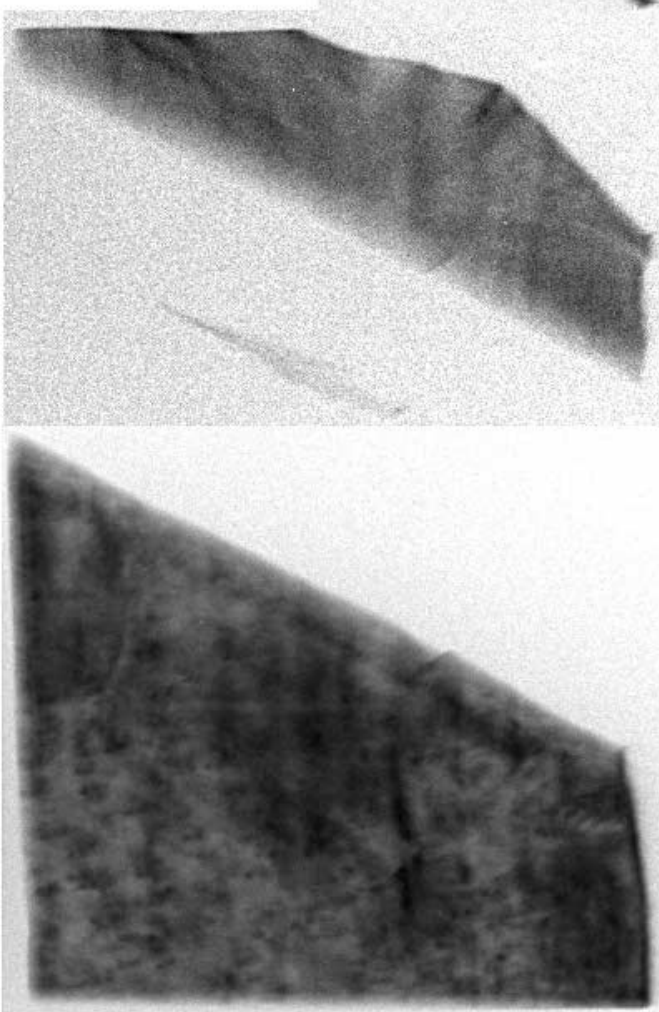

Fig. 8. IR map, gamma map, and topograph details for a $27 \mathrm{~mm} \times 27 \mathrm{~mm} \times 2 \mathrm{~mm}$ CZT crystal. 
a)

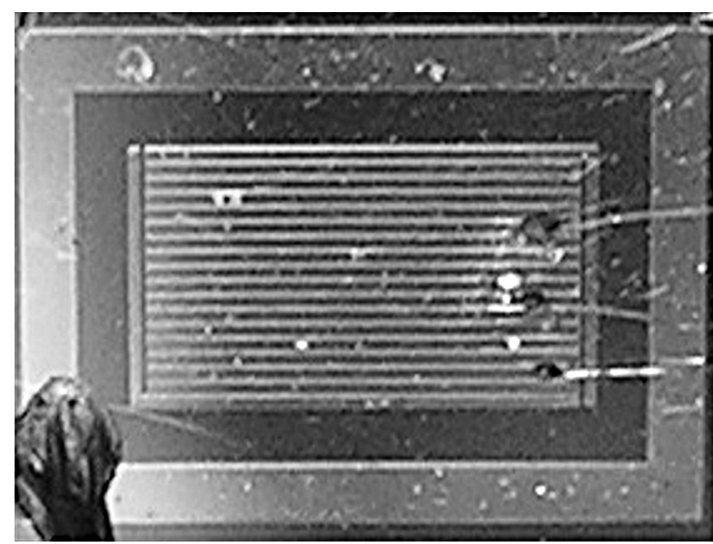

C)
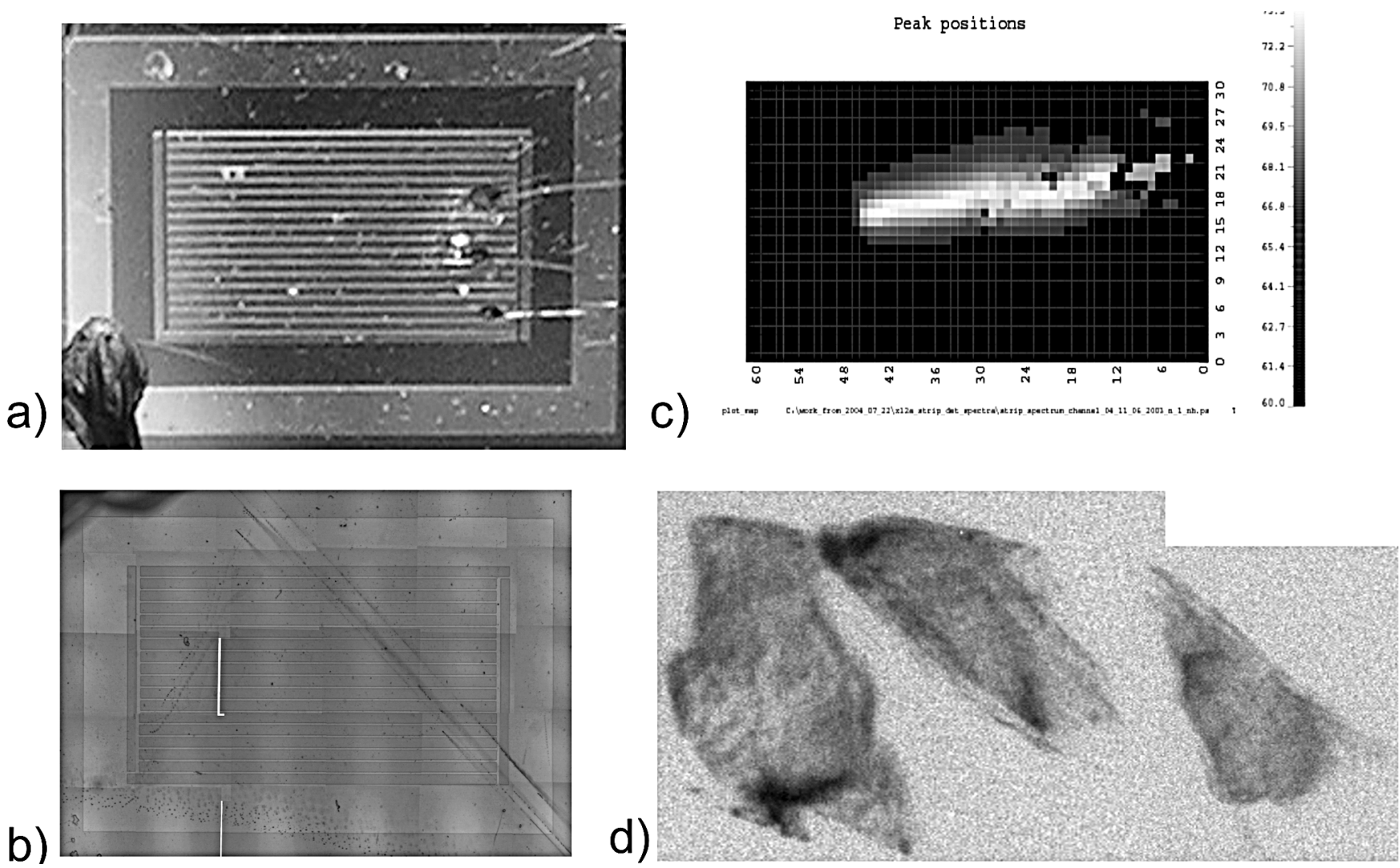

Fig. 9. (a) Strip detector on its readout board.

(b) IR mapping before

visualization of the complete sample simultaneously, with high resolution, but it suffers from limited sensitivity to weak distortions, as in our case a single defect in the bulk. To understand defect distribution, thinner (100's of micrometers) samples should be studied.

Results from these studies and IR and gamma mapping can be correlated, as shown in the two following examples. ${ }^{14-17}$

In Fig. 8, a gamma response map, a transmission IR map, and topographs of a $27 \mathrm{~mm} \times 27 \mathrm{~mm} \times 2 \mathrm{~mm}$ CZT crystal are shown. The gamma map of the crystal in planar configuration is acquired by raster scanning the detector with a collimated source and recording data through a multi-channel analyzer (MCA). From the spectrum, the photopeak position gives a measurement of the response pulse height. Its variation across the map exhibits the uniformity of the response all over the detector area. Along the grain boundary, the crystal shows a larger conductivity, which alters the internal electric field. Furthermore, spatial variations in trap density cause spatial variations in charge transport properties. These nonuniformities locally affect the response of the detector. A strong correlation between poor detector response and tellurium decorated grain boundaries, clear in the IR map, can be observed. Evidently, topographs show a remarkable correspondence with IR and gamma maps. In the topographs, we can observe several domains. Each domain corresponds to a region exhibiting uniform gamma response, separated by grain boundaries. Contrasts inside each domain should be related to the tellurium inclusions.

In Fig. 9, maps related to a crystal of $6 \mathrm{~mm} \times$ $4 \mathrm{~mm} \times 2 \mathrm{~mm}$ are shown. This crystal was used to fabricate a strip detector, 16 strips of $4 \mathrm{~mm} \times 100 \mu \mathrm{m}$ with $125 \mu \mathrm{m}$ pitch. To identify defect positions, a transmission IR image was carried out after lithography, just before gold deposition (linear absorption coefficient for gold is a few nanometers in the IR field). As the depth of the focus of the IR optical system is a few tens of micrometers, defects seen in this image are those in the first $\sim 30 \mu \mathrm{m}$ depth. This map exhibits a long twin boundary on the centerright side; a region with high concentration of $\mathrm{Te}$ precipitates in the lower left corner; and another inclusions-rich region just above the region previously described. Three IR maps are performed at three focal planes related to the top, the middle, and the bottom of the crystal, showing the twin boundary through the bulk. This could be the reason why this detector cannot sustain a bias voltage higher than $270 \mathrm{~V}$. A $500 \mathrm{~V}$ voltage is normally applied for a 2 -mm-thick detector. An x-ray map of the strip, crossing the twin boundary, was performed at the $\mathrm{X} 12 \mathrm{~A}$ beamline, using a $25 \mu \mathrm{m} \times 25 \mu \mathrm{m}$ beam size with $30 \mathrm{keV}$ energy and $50 \mu \mathrm{m}$ step. As for the gamma map, a raster scan was performed and data were recorded through an MCA. The peak position, evaluated from Gaussian fitting of the spectra, showed nonuniformity in the response of the strip. 
The drop of performance along the twin boundary is obviously related to localized enhanced charge loss, typical along macroscopic crystal defects. The effect of the twin on the charge collection efficiency becomes clearer when the bias voltage is decreased. These results are confirmed by WBXT performed all over the detector. The twin boundary (seen on the IR map) separates the two "triangular"-shaped domains.

\section{CONCLUSIONS}

High-energy transmission x-ray diffraction techniques have been successfully applied to investigate CZT crystal quality. These are nondestructive and high-resolution techniques suitable for bulk and in-situ measurements. An interesting correlation with results achieved from other techniques, used to characterize material qualified for detector fabrication, was shown. More systematic studies are required to improve CZT quality needed to enhance detector performance.

\section{ACKNOWLEDGEMENTS}

The authors gratefully acknowledge the support of all the staff, technicians, and scientists of the National Synchrotron Light Source, particularly Wolfgang Caliebe, Zhijian Yin, and Jean Jakoncic. Appreciation goes to the U.S. Department of Energy for the support provided by LDRD funding. Use of the NSLS was supported by U.S. Department of Energy Contract No. DE-AC02-76CH00016.

\section{REFERENCES}

1. J.F. Butler, C.L. Lingren, and F.P. Doty, IEEE Trans. Nucl. Sci. 39, 605 (1992).

2. H. Hermon et al., Nucl. Instrum. Methods Phys. Res. A 410, 100 (1998).

3. F.P. Doty, J.F. Butler, J.F. Schetzina, and K.A. Bowers, J. Vac. Sci. Technol. B 10, 1418 (1992).

4. J.M. Van Scyoc, B.A. Brunett, H. Yoon, T.S. Gilbert, N.R. Hilton, J.C. Lund, and R.B. James, Nucl. Instrum. Methods A 428, 1 (1999).

5. B.H. Parker, C.M. Stahle, S.D. Barthelmy, A.M. Parsons, J. Tueller, J.T. Van Sant, B.F. Munoz, S.J. Snodgrass, and R.E. Mullinix, SPIE 3768, 129 (1999).

6. B.K. Tanner, X-ray Diffraction Topography (Oxford, England: Pergamon Press, 1976), pp. 1-174.

7. M. Dudley, "X-ray Topography," NSLS Annual Report (1991, 1993).

8. G.D. Yao, M. Dudley, T. Fanning, and D. Larson, NSLS Annual Report (1991).

9. M. S. Goorsky, H. Yoon, M. Ohler, and K. Liss, SPIE 3768, 108 (1999).

10. K. Garg, M. Srivastava, R.C. Narula, R.K. Bagai, and V. Kumar, J. Cryst. Growth 260, 148 (2004).

11. Z. Zhong, C.C. Kao, D.P. Siddons, and J.B. Hastings, J. Appl. Cryst. 34, 504 (2001).

12. Z. Zhong, C.C. Kao, D.P. Siddons, and J.B. Hastings, J. Appl. Cryst. 34, 646 (2001).

13. http://www.certif.com.

14. M. Shieber, T.E. Schlesinger, R.B. James, H. Hermon, H. Yoon, and M. Goorshky, J. Cryst. Growth 237-239, 2082 (2002).

15. B.A. Brunett, J.M. Van Scycoc, T.E. Schlesinger, and R.B. James, Nucl. Instrum. Methods A 428, 76 (2001).

16. G.S. Camarda, E.M. Kakuno, G. Carini, A.E. Bolotnikov, G.W. Wright, R.B. James, and D.P. Siddons (Paper presented at IEEE Proc., Portland, OR, 2003).

17. G.S. Camarda, G. Carini, Z. Zhong, D.P. Siddons, A.E. Bolotnikov, G.W. Wright, and R.B. James, SPIE 5540 (2004). 